\title{
BOTT-TYPE SYMPLECTIC FLOER COHOMOLOGY AND ITS MULTIPLICATION STRUCTURES
}

\author{
Yongbin RuAN And GANG Tian
}

One of the celebrated theories in the 80's is the Floer (co)-homology. Floer first defined his cohomology in the context of symplectic geometry in an attempt to solve the Arnold conjecture. Later, he extended his cohomology to gauge theory, which he called Instanton cohomology. Even though the gauge theoretic Floer (co)-homology was an analogue of his symplectic Floer (co)-homology, it has attracted more attention during last few years and experienced a rapid development, due to the work of Donaldson, Taubes, Kronheimer, Mrowka, Stern, Finturshel, Fukaya, Austin-Braam and other gauge theorists. The development of the symplectic Floer (co)homology was at a slower pace. Recently, partly inspired by the quantum cohomology theory, there is a surge of interest in studying multiplication structures on the symplectic Floer cohomology. It seems that those multiplications can be unified by introducing a Bott-type symplectic Floer cohomology. The main purpose of this paper is to give a brief description of the construction of the Bott-type symplectic Floer cohomology and its multiplication structures on semi-positive symplectic manifolds. The details of the proof will appear in a forthcoming paper. The gauge theoretic Bott-type Floer homology (without considering the multiplication ) has been previously established by Austin-Braam $[\mathrm{AB}]$ and Fukaya $[\mathrm{Fu}]$, based on the analysis developed by Mrowka $[\mathrm{M}]$. We are partially inspired by their work. We should point out that these multiplication structures are unique for the symplectic Floer cohomology and there is no analogue in gauge theory.

The quantum cohomology played a critical role in recent developments in algebraic geometry and mathematical physics. Recently, we established its mathematical foundation for semi-positive symplectic manifolds [RT]. The quantum cohomology imposed a new ring structure on the ordinary cohomology with the Novikov ring as its coefficient ring. The new multiplication is called quantum multiplication. Such a quantum cohomology captures the full information of Witten's topological sigma model theory.

Received December 19, 1994. 
The symplectic Floer cohomology was defined for a hamiltonian symplectomorphism whose fixed points are nondegenerate. A crucial step to a solution of the Arnold conjecture is to show that the Floer cohomology is the same as the ordinary cohomology. Furthermore, Floer also introduced a multiplication on the Floer cohomology, extrinsic multiplication, by the ordinary cohomology. This multiplication is very useful in the resolution of the Arnold conjecture in the case of degenerate fixed points $[\mathrm{F}],[\mathrm{OV}]$. On the other hand, there is a natural way to define an intrinsic multiplication on the Floer cohomology by using the perturbed $J$-holomorphic maps from a pair-pants to the symplectic manifold. In fact, such an intrinsic multiplication has been established by Betz and Rade [BR]. It has been conjectured that all these multiplications (intrinsic and extrinsic) are the same as the quantum multiplication. For the extrinsic multiplication, a physical argument was given by Sadov $[\mathrm{S}]$. In [P], Piunikhin outlined a different approach towards the proof of the equivalence of the extrinsic multiplication and the quantum multiplication by using intersection theory. The difficulty with this approach lies in the fact that the compactification of the moduli space of trajectories has codimension 1 boundary (cf. section 2 ). On the other hand, the equivalence of the intrinsic multiplication and the quantum multiplication has not been addressed at all.

A hamiltonian symplectomorphism is Bott-type if and only if the fixedpoint set consists of nondegenerate submanifolds in the sense of Bott (cf. Definition 1.1). It is easy to see that identity and nondegenerate hamiltonian symplectomorphisms are Bott-type. The ordinary Floer cohomology was defined in terms of a nondegenerate hamiltonian symplectomorphism. But almost all the analysis in this case fails when we replace the nondegenerate symplectomorphism by a Bott-type symplectomorphism. Topologically, our Bott-type Floer cohomology is defined as the cohomology of a chain complex consisting of all geometric chains (cf. section 2) in the fixed-point set of the Bott-type symplectomorphism. In order to define the boundary operator on such a chain complex, we have to prove that the compactification of the moduli space of trajectories is a simplicial complex (cf. Theorem 2.1). It involves a very difficult analysis of the behavior of the moduli space of trajectories near degenerations. This analysis refines that developed in $[\mathrm{RT}]$. We will show that our Bott-type Floer cohomology is actually independent of a particular Bott-type hamiltonian symplectomorphism. Our Bott-type cohomology can often be interpreted as the limit of a spectral sequence. Such a spectral sequence arises from the filtration of our chain complex. As a corollary of our Bott-type Floer cohomology theory, we prove a version of the Arnold conjecture for Bott-type hamiltonian symplectomorphisms (cf. Corollary 1.3).

Furthermore, we will construct two different multiplications on our 
Bott-type Floer cohomology (extrinsic and intrinsic) and prove that they are independent of a particular Bott-type hamiltonian symplectomorphism. It is easy to check that the intrinsic and extrinsic multiplications of identity are the same as the quantum multiplication. Therefore, the multiplications on the ordinary Floer homology of nondegenerate hamiltonian symplectomorphisms are the same as the quantum multiplication. In the last section, we define the quantum Massey product. The same method can be used to define various secondary operations over the Bott-type Floer cohomology (cf. section 5), generalizing Fukaya's construction [Fu1]. A famous theorem in algebraic topology states that the ordinary Massey product vanishes for Kahler manifolds. Our approach indicates that the quantum Massey product should also vanish for Kahler manifolds. Our Bott-type Floer cohomology also gives a new approach to calculating the quantum cohomology of fibration, which is a rather difficult problem in algebraic geometry. We shall leave it for the future research.

During the preparation of this paper, Piunikhin, Salamon and Schwarz also announced an independent proof of the equivalence of the multiplications on the Floer cohomology and the quantum multiplication. Their approach is completely different from ours.

\section{Statements of main theorems}

Let $(V, \omega)$ be a closed symplectic manifold. Given any function $H$ on $V \times S^{1}$, we can associate a vector field $X_{H}$ as follows:

$$
\omega\left(X_{H}(z, t), v\right)=v(H)(z, t), \text { for any } v \in T_{z} V
$$

We call $H$ a periodic hamiltonian and $X_{H}$ a hamiltonian vector field associated to $H$. Let $\phi_{t}(H)$ be the integral flow of the hamiltonian vector field $X_{H}$. Then $\phi_{1}(H)$ is a hamiltonian symplectomorphism.

Definition 1.1. We declare a periodic hamiltonian $H$ to be of Bott-type if and only if the fixed-point set $F\left(\phi_{1}(H)\right)$ of $\phi_{1}(H)$ is nondegenerate in the sense of Bott, i.e., $F\left(\phi_{1}(H)\right)$ consists of smooth submanifolds in $V$ and $\phi_{1}(H)$ is nondegenerate in the normal directions of these submanifolds.

Example 1.2. If $H$ is nondegenerate, then $H$ is of Bott-type. If $H=0$, then $\phi_{1}(H)$ is the identity and clearly of Bott-type, so $H=0$ is of Botttype.

To state the main theorems, we need some notations. Let $H$ be a Botttype periodic hamiltonian function. Let $\mathcal{L}(V)$ be the space of contractible maps (sometimes called contractible loops) from $S^{1}$ into $V$ and $\tilde{\mathcal{L}}(V)$ be 
the universal covering of $\mathcal{L}(V)$; namely, $\tilde{\mathcal{L}}(V)$ is as follows:

$$
\tilde{\mathcal{L}}(V)=\left\{(u, x) \mid u \in \mathcal{L}(V), x: D^{2} \rightarrow V \text { such that } u=\left.x\right|_{\partial D^{2}}\right\} / \sim,
$$

where the equivalence relation $\sim$ is the homotopic equivalence of $x$. The covering group of $\tilde{\mathcal{L}}$ over $\mathcal{L}$ is $\pi_{2}(V)$. We can define a symplectic action functional on $\tilde{\mathcal{L}}(V)$,

$$
a_{H}((u, x))=\int_{D^{2}} x^{*} \omega+\int_{0}^{2 \pi} H(t, u(t)) d t
$$

It follows from the closedness of $\omega$ that $a_{H}$ descends to the quotient space by $\sim$. The Euler-Lagrange equation of $a_{H}$ is

$$
\dot{u}-X_{H}(t, u(t))=0
$$

Let $R(H)$ be the critical point set of $a_{H}$, i.e., the set of smooth contractible loops satisfying the Euler-Lagrange equation. Using that $H$ is Bott-type, one can show that $R(H)$ is a union of smooth manifolds and $a_{H}$ is nondegenerate on the normal bundle of $R(H)$ in $\tilde{\mathcal{L}}(V)$. Though the image of $R(H)$ in $\mathcal{L}(V)$ is a finite union of compact smooth manifolds, $R(H)$ may have infinitely many connected components, which are generated by the covering transformation group $\pi_{2}(V)$.

Given $(x, u) \in R(H)$, choose a symplectic trivialization

$$
\Phi(t): R^{2 n} \rightarrow T_{x(t)} V
$$

of $x^{*} T V$ which extends over the disc $D$. Linearizing the Hamiltonian differential equation along $x(t)$, we obtain a path of symplectic matrices

$$
A(t)=\Phi(t)^{-1} d \phi_{t}(x(0)) \Phi(0) \in S p(2 n, R) .
$$

Here the symplectomorphism $\phi_{t}: V \rightarrow V$ denotes the time- $t$-map of the hamiltonian flow

$$
\dot{\phi}_{t}=\nabla H_{t}\left(\phi_{t}\right) \text {. }
$$

Then, $A(0)=I d$ and $A(1)$ is conjugate to $d \phi_{1}(x(0))$. In the Bott-type case, 1 could be the eigenvalue of $A(1)$. In this case, we perturb the path $A(t)$ slightly to $\tilde{A(t)}$ such that 1 is not an eigenvalue of $\tilde{A(1)}$. Then, we can assign a Conley-Zehnder index for $\tilde{A(t)}$. The Conley-Zehnder index of $(x, u)$ is defined as the sum of the Conley-Zehnder index of path $\tilde{A(t)}$ and the number of 1-eigenvalues of $A(1)$ which becomes negative in the perturbation. The Conley-Zehnder index is independent of the choice of the trivialization and the perturbations. Furthermore, it is constant on a connected component of $R(H)$. Then, we can decompose $R(H)$ as

$$
R(H)=\cup_{i} R_{i}(H),
$$


where $R_{i}(H)$ consists of critical points in $R(H)$ with the Conley-Zehnder index $i$.

The group $\Gamma_{0}=\left\{A \in \pi_{2}(V) ; C_{1}(V)(A)=0\right\}$ acts on $\tilde{\mathcal{L}}(V)$ and generates (possibly) infinitely many components in $R_{i}$. We denote by $R_{i}^{s}(H)$ the component of $R_{i}(H)$, then $R_{i}(H)=\bigcup R_{i}^{s}(H)$. To define the Bott-type Floer cohomology, we first construct a chain complex and boundary map $\left(C^{*}(V, H), \delta^{H}\right)$. The detailed construction will be given in the next section. Roughly speaking, $C^{m}(V, H)$ consists of $m-i$ cochains of $R_{i}$ for all $i$. The boundary map $\delta^{H}$ involves the trajectory spaces of all the dimensions.

We define the Bott-type Floer cohomology $H F^{*}(V, H)$ to be the cohomology of the chain complex $\left(C^{*}(V, H), \delta^{H}\right)$ (cf. Definition 2.7) It follows from a standard argument that this Bott-type Floer cohomology $H F^{*}(V, H)$ is independent of any particular Bott-type hamiltonian function $H$ (Lemma 2.8). If $H$ satisfies an additional condition of self-indexing (cf. section 2), let $E_{i, j}^{1}$ to be the set of sum $f=\sum_{s} f_{s}$, where $f_{s} \in$ $\operatorname{Hom}\left(H_{j}\left(R_{i}^{s}(H), Z\right), Z\right)$ satisfying the following condition

$$
\left\{s \mid f_{s} \neq 0, a_{H}\left(R_{i}^{s}\right) \geq C\right\}<\infty
$$

where $C$ is any constant.

Proposition 1.1. Let $(V, \omega)$ be a semi-positive symplectic manifold and $H$ be a self-indexing Bott-type hamiltonian. Then there exists a spectral sequence $E_{* *}^{*}$ on the upper half plane where $E_{i, j}^{1}$ is defined as above and $E_{i, j}^{*} \Longrightarrow H F^{i+j}(V, H)$.

It is easy to check that both nondegenerate hamiltonian functions and the zero hamiltonian function are self-indexing.

We recall that the Novikov ring $\Lambda_{\omega}$ is defined as follows: each $a$ in $H_{2}(V, Z)$ induces a homomorphism, formally denoted by $e^{2 \pi \sqrt{-1} a}$, from $H^{2}(V, C)$ into $C^{*}$ by sending $\phi$ to $e^{2 \pi \sqrt{-1} \phi(a)}$, then the Novikov ring $\Lambda_{\omega}$ is the ring of the formal series of the form

$$
\lambda=\sum_{a} \lambda_{a} e^{2 \pi \sqrt{-1} a}
$$

such that for each $c>0$, the number of nonzero $\lambda_{a}$ with $\omega(a) \leq c$ is finite.

The following lemma can easily be proved. In fact, if the hamiltonian function $H$ is identically zero, the spectral sequence $E_{*, *}^{*}$ defined in Proposition 1.1 degenerates at $E_{*, *}^{1}$ (cf. section 2).

Lemma 1.2. As a $\Lambda_{\omega}$-module, the cohomology $H F^{*}(V, 0)=H^{*}\left(V, \Lambda_{\omega}\right)$, i.e., the ordinary cohomology with the coefficient ring $\Lambda_{\omega}$. 
It follows from this lemma that the Bott-type Floer cohomology $H F^{*}(V)$ is the same as the ordinary cohomology with the coefficient ring $\Lambda_{\omega}$. In the nondegenerate case, this was proved by Floer by using a time independent, generic Morse funtion. His proof involved some delicate analysis on the contributions of non-trivial periodic orbits. The proof here is more direct.

Let $F(H)=\cup_{i} F_{i}(H)$ be the fixed point set of $H$, where $F_{i}(H)$ are the connected components. Clearly, $F(H)$ is just the quotient of $R(H)$ by the covering transformation group $\pi_{2}(V)$. If $X$ is a topological space, we use $B(X)$ to denote the sum of the Betti numbers. With some additional work, we can prove

Theorem 1.3. Let $(V, \omega)$ br a semi-positive symplectic manifold. Then

$$
\sum_{i} B\left(F_{i}(H)\right) \geq B(V)
$$

This can be considered as the resolution of a generalized Arnold conjecture for Bott-type hamiltonian symplectomorphisms.

Theorem 1.4. Let $H$ be a Bott-type hamiltonian. Any $\alpha \in H^{*}(V, Z)$ induces an natural action on $H F_{*}(V, H)$. Furthermore, the action is independent of $H$, so there is an action of $H^{*}(V, Z)$ on $H F^{*}(V)$.

It is not hard to see that when $H$ is nondegenerate, the action of $\alpha$ is the same as that of Floer. The following is a simple observation.

Lemma 1.5. When $H=0$, the action of $\alpha$ is the same as the quantum multiplication if we consider $\alpha$ as an element of the quantum homology.

Theorem 1.6. There is a multiplication

$$
h: C^{i}(V, H) \otimes C^{j}(V, H) \rightarrow C^{i+j}(V, H)
$$

such that

$$
\delta^{H} h(x \otimes y)=h\left(\delta^{H} x \otimes y\right)+(-1)^{i+j}\left(x \otimes \delta^{H} y\right) .
$$

Hence, it induces an intrinsic multiplication on the Bott-type Floer homology $H^{*}$. Furthermore, this multiplication is independent of $H$.

As before, one can show that when $H$ is nondegenerate, this intrinsic multiplication on $H F^{*}(V, H)$ is the same as the intrinsic multiplication on the Floer homology. The following lemma can be easily proved.

Lemma 1.7. When $H=0$, the intrinsic multiplication is the same as the quantum multiplication. 
It follows from Lemma 1.6, 1.7

Theorem 1.8. The extrinsic, intrinsic and quantum multiplications on $H^{*}\left(V, \Lambda_{\omega}\right)$ are the same.

In the next three sections, we shall describe the construction of our Bott-type Floer cohomology and the proof of Theorems 1.4, 1.6. In the last section, we define the Massey product. In fact, one can define more complicated secondary multiplications in a similar fashion.

\section{Bott-type symplectic Floer cohomology}

Fix a periodic compactible almost complex structure $J$, i.e., $J=J_{t}$ is a circle family of compactible almost complex structures. We can consider the gradient flow equation of $a_{H}$ :

$$
\mathcal{F}(u(s, t))=\frac{\partial u}{\partial s}+J(t, u) \frac{\partial u}{\partial t}+\nabla H(t, u)=0,
$$

where we use $s$ to denote the time variable and $t$ to denote the circle variable in a traditional way. Let

$$
\begin{aligned}
\tilde{\mathcal{M}}=\left\{u: S^{1}\right. & \times R \rightarrow R \mid \\
\mathcal{F}(u) & \left.=0, \int_{S^{1} \times R}\left(\left|\frac{\partial u}{\partial s}\right|^{2}+\left|J(t, u) \frac{\partial u}{\partial t}+\nabla H(t, u)\right|^{2}\right) d s d t<\infty\right\} .
\end{aligned}
$$

Analytically, there is a difference between our Bott-type case and Floer's case. Since the set of critical points is not discrete, in general we don't necessarily have a flow line from or to a critical point. In fact, it is not clear that a flow line will converge to a point in any critical manifolds at all. For example, in prior, a flow line could spiral into a critical submanifold, and its limit set would be a circle instead of a point. The flow line may also converge to a critical point at a very slow speed. The following lemma rules out this phenomenon for Bott-type hamiltonian functions.

Lemma 2.1. For every $u \in \tilde{\mathcal{M}}, u_{s}(t)=u(s, t)$ converges to $u_{ \pm \infty}(t) \in$ $R(H)$ when $s \rightarrow \pm \infty$. If $H$ is of the Bott-type, $u_{s}$ converges exponentially to its limit, i.e., $\left|u_{s}-u_{ \pm \infty}\right|<C e^{-\delta|s|}$ for $s \geq|T|$.

This can be proved by a standard argument in stability theory. The key point is that all critical points of $H$ are integrable, since $H$ is of Bott-type. The exponential decay serves as the basis for the transversality theory. It may fail for a hamiltonian $H$ of non-Bott type. In the gauge theory, such examples had been found by Mrowka [M]. 
By this lemma, we can divide $\tilde{\mathcal{M}}$ into

$$
\tilde{\mathcal{M}}=\bigcup \tilde{\mathcal{M}}(i, j)
$$

where

$$
\tilde{\mathcal{M}}(i, j)=\left\{u \in \tilde{\mathcal{M}} ; \lim _{s \rightarrow-\infty} u_{s} \in R_{i}(H), \lim _{s \rightarrow \infty} u_{s} \in R_{j}(H)\right\} .
$$

Clearly, $R^{1}$ acts on $\tilde{\mathcal{M}}$ as translations in time. Let $\mathcal{M}=\tilde{\mathcal{M}} / R^{1}$ and $\mathcal{M}(i, j)=\tilde{\mathcal{M}}(i, j) / R^{1}$.

Lemma 2.2. For a generic $J, \mathcal{M}(i, j)$ is a smooth, oriented manifold of dimension $i-j-1+\operatorname{dim} R_{i}(H)$.

By Lemma 2.1, we can define the boundary maps

$$
\partial_{-}^{i}: \mathcal{M}(i, j) \rightarrow R_{i}(H), \partial_{+}^{j}: \mathcal{M}(i, j) \rightarrow R_{j}(H)
$$

For a generic $J$, the boundary maps $\partial_{ \pm}^{i}$ are smooth and transverse to each other whenever they have the same target space.

Now we are ready to construct the chain complex and boundary maps.

Definition 2.3 ( $[\mathrm{Fu}])$. A finite simplicial complex $P$ is said to be an abstract geometric chain of dimension $n$ if

(1) There is a subset $P_{\text {reg }}$ which is an oriented manifold of dimension $n$ with boundary $\partial P_{\text {reg }}$.

(2) We put $P_{s}=P-P_{\text {reg. }}$. Then, $P_{s}$ is a subcomplex of $P$.

(3) $\operatorname{dim} P_{s} \leq n-2$.

(4) We put $\partial P=\overline{\partial P_{\text {reg }}}$. Then, $\operatorname{dim} P_{s} \cap \partial P \leq n-3$.

The following theorem contains the most technical and difficult work of this paper.

Theorem 2.4. The moduli space $\overline{\mathcal{M}(i, j)}$ admits a compactification $\overline{\mathcal{M}}(i, j)$, which is an abstract geometric chain.

For a smooth manifold, a pair $(P, f)$ of an abstract geometric chain $P$ and a continuous map $f: P \rightarrow X$ is said to be a geometric chain of $X$, if $f$ is piecewise smooth and the restrictions of $f$ to $P_{\text {reg }}$ and $\partial P_{\text {reg }}$ are smooth. For each component $R_{i}^{s}(H)$, let $C_{m}^{t}\left(R_{i}^{s}(H), Z\right)$ be the space of geometric chains transversal to all the boundary maps into $R_{i}^{s}(H)$. It follows from the standard transversality theory that

$$
H_{*}\left(C^{t}\left(R_{i}^{s}(H), \partial\right)\right)=H_{*}\left(R_{i}^{s}(H), Z\right)
$$


where $\partial$ is the boundary map of the ordinary homology. In order to define the boundary operator, we have to extend both $\partial^{+}$and $\partial^{-}$to the compactification of $\mathcal{M}(i, i-k)$.

Lemma 2.5. For any $i$ and $j$, both $\partial^{+}$and $\partial^{-}$can be extended to be continuous maps from the compactification $\overline{\mathcal{M}}(i, j)$. We will use the same symbols to denote the extensions.

Intuitively, these extensions are defined as follows: let $u_{\alpha}$ be any sequence in $\overline{\mathcal{M}}(i, j)$, which converges to $u_{\infty}$ in $\overline{\mathcal{M}}(i, j) \backslash \mathcal{M}(i, j)$. Then $u_{\infty}$ is a union of $u_{\infty, \beta}$ in $\overline{\mathcal{M}}\left(i_{\beta}, j_{\beta}\right)$, where $1 \leq \beta \leq \ell, i_{1}=i, j_{\ell}=j$, and there are finitely many pseudo-holomorphic spheres. We simply define $\partial^{+}\left(u_{\infty}\right)=\partial^{+}\left(u_{\infty, \ell}\right)$ and $\partial^{-}\left(u_{\infty}\right)=\partial^{-}\left(u_{\infty, 1}\right)$. One can show that these extensions are continuous with respect to the Gromov-Uhlenbeck topology on $\overline{\mathcal{M}}(i, j)$. Now we can define a boundary map

$$
\left.\partial_{k}: C_{m}^{t}\left(R_{i}(H)\right), Z\right)=\oplus_{s} C_{m}^{t}\left(R_{i}^{s}(H), Z\right) \rightarrow C_{m+k-1}^{t}\left(R_{i-k}, Z\right)
$$

as follows: for any $(P, f) \in C_{m}^{t}\left(R_{i}^{s}, Z\right)$, the fiber product

$$
P \times_{R_{i}^{s}(H)} \overline{\mathcal{M}}(i, i-k)
$$

is an abstract geometric chain of dimension $m+k-1$. This can be shown by taking simplicial approximations of $\partial^{-}$. Then $\partial_{k}(C)$ is the composition of the natural projection $P \times_{R_{i}^{s}(H)} \overline{\mathcal{M}}(i, i-k) \rightarrow \overline{\mathcal{M}}(i, i-k)$ and the boundary map $\partial^{+}: \overline{\mathcal{M}}(i, i-k) \rightarrow R_{i-k}$. In general, even if $C$ is an honest chain, $\partial_{k}(C)$ could have infinitely many components involving infinitely many components of $R_{i}$. We define the cochain complex $C_{t}\left(R_{i}(H), Z\right) \subset$ $\oplus_{s} \operatorname{Hom}\left(C^{t}\left(R_{i}^{s}(H), Z\right), Z\right)$ satisfying the finiteness condition described in the previous section. We define the coboundary map

$$
\delta_{k}: C_{t}^{m}\left(R_{i}(H), Z\right) \rightarrow C_{t}^{m-k+1}\left(R_{i+k}(H), Z\right)
$$

as usual, i.e., $\delta_{k}(\alpha)=\alpha \partial_{k}$. It follows from the Gromov-Uhlenbeck compactness theorem that the image of $\delta_{k}$ satisfies the finiteness condition (1.5). Furthermore, we define $\partial_{0}=(-1)^{n+j} \partial$ and $\delta_{0}$ similarly.

We put

$$
C_{t}^{n}(V, H)=\oplus_{i} C_{t}^{n-i}\left(R_{i}(H), C\right), \delta^{H}=\oplus \delta_{k}
$$

Lemma 2.6. $\left(C_{t}^{n}(V, H), \delta^{H}\right)$ is a chain complex with the boundary map $\delta^{H}$, namely, $\delta^{H} \delta^{H}=0$.

Definition 2.7. The Bott-type Floer cohomology $H F^{*}(V, H)$ for the hamiltonian function $H$ is defined to be the cohomology $H^{*}\left(C_{t}(V, H), \delta^{H}\right)$. 
Lemma 2.8. The Bott-type Floer cohomology

$$
H F^{*}(V, H)=H^{*}\left(C_{t}(V, H), \delta^{H}\right)
$$

is independent of $H$.

Because of this, we sometimes write $H F^{*}(V)$ for $H F^{*}(V, H)$.

To prove Theorem 1.3, one constructs an injective map

$$
\pi: \operatorname{Ker} \delta_{H} \rightarrow \oplus \operatorname{Ker} \delta_{0}
$$

where the restriction of $(-1)^{n+i} \delta_{0}$ to each $R_{i}$ is the standard coboundary operator. This map induces an injective homomorphism from $H F^{*}(V, H)$ into the quotient of $\oplus H^{*}\left(R_{i}, Z\right)$ by $\pi\left(\operatorname{Im}\left(\delta_{H}\right)\right)$ as the modules over the Novikov rings. Therefore, we prove Theorem 1.3.

Definition 2.9. $H$ is called self-indexing if for a generic $J, \mathcal{M}(i, j)=\emptyset$ for $j \geq i$.

Clearly, if $H$ is nondegenerate, $H$ is self-indexing. Furthermore, $H=0$ is self-indexing. When $H$ is self-indexing, there is a filtration

$$
\mathcal{F}_{k}\left(C_{t}^{n}(V, H)\right)=\oplus_{i \geq k} C_{t}^{n-i}\left(R_{i}\right) .
$$

By standard homological algebra theory, such a filtered chain complex $\left(C_{t}^{n}(V, H), \delta^{H}\right)$ induces a spectral sequence convergent to

$$
H^{*}\left(C_{t}(V, H), \delta^{H}\right)
$$

Moreover, its $E_{i, j}^{1}$ term equals $H^{j}\left(F_{k} / F_{k-1}\right)$, which is the subspace of elements in $H^{j}\left(R_{i}, Z\right)=\oplus_{s} H^{j}\left(R_{i}^{s}(H), Z\right)$, which satisfy the finiteness condition (1.5). Then, Proposition 1.1 follows from the filtration.

When $H$ is nondegenerate in the classical sense, the corresponding spectral sequence degenerates at $E^{2}$; it follows from the definition that our Bott-type Floer cohomology is just the Floer cohomology in the ordinary sense. When $H=0$, it is easy to check that $H F^{*}(V, 0)$ is the same as $H^{*}\left(V, \Lambda_{\omega}\right)$, where $\Lambda_{\omega}$ is the Novikov ring.

\section{The extrinsic multiplication}

In $[F]$, Floer defined a multiplication on the Floer cohomology by the ordinary cohomology (extrinsic multiplication). Then, he used the extrinsic multiplication to solve some cases of the Arnold conjecture where the fixed points are degenerate. We should point out that Floer did not define the extrinsic multiplication directly for degenerate hamiltonian symplectomorphisms. He only defined it for nondegenerate hamiltonian symplectomorphisms and studied its asymptotic behavior when hamiltonians function 
become degenerate. We now generalize Floer's construction to the Botttype case. We need to construct an action of $H^{*}(V, Z)$ on $H F^{*}(V, H)$. For this purpose, we will construct an action of generic cocycles in $V$ on $\left(C_{t}(V, H), \delta^{H}\right)$.

Given $\kappa \in C_{t}^{m-i}\left(R_{i}(H), Z\right)$ and a generic cocycle $\alpha$ representing a cohomology class $[\alpha] \in H^{k}(V, Z)$, define

$$
\alpha(\kappa) \in C_{t}^{m+k}(V, H)=\oplus_{j} C^{m+k-j}\left(R_{j}, Z\right)
$$

as follows:

Let $\alpha(\kappa)=\sum_{j} \alpha^{j}(\kappa)$, where $\alpha^{j}(\kappa) \in C_{t}^{m+k-j}\left(R_{j}, Z\right)$. Consider the evaluation map

$$
e: \tilde{\mathcal{M}}(j, i) \rightarrow V
$$

by $e(u)=u(0,0)$. Recall that modulo bubbling offs, the boundary of $\overline{\mathcal{M}}(j, i)$ consists of broken trajectories

$$
\tilde{\mathcal{M}}\left(j, k_{1}\right) \times \tilde{\mathcal{M}}\left(k_{1}, k_{2}\right) \times \cdots \times \tilde{\mathcal{M}}\left(k_{s}, i\right) .
$$

The evaluation map extends over such a compactification. Since the evaluation map only takes value on a component, it descends to the quotient where we divide all but one component by time translations. In other words, we can get a compactification whose boundary consists of

$\mathcal{M}\left(j, k_{1}\right) \times \cdots \times \mathcal{M}\left(k_{p-1}, k_{p}\right) \times \tilde{\mathcal{M}}\left(k_{p}, k_{p+1}\right) \times \mathcal{M}\left(k_{p+1}, k_{p+2}\right) \times \cdots \times \mathcal{M}\left(k_{s}, i\right)$.

We denote this smaller compactification by $\overline{\mathcal{M}}^{*}(j, i)$. Then $e$ extends over $\overline{\mathcal{M}}^{*}(j, i)$.

Lemma 3.1. $\overline{\mathcal{M}}^{*}(j, i)$ is an abstract geometric chain.

This can be proved by the same arguments as those in the proof of Theorem 2.4.

For any geometric chain $(P, f) \in C_{m+k-j}^{t}\left(R_{j}^{s}(H), Z\right)$, the fiber product

$$
P \times_{R_{j}^{s}} \overline{\mathcal{M}}^{*}(j, i)
$$

is an abstract geometric chain of dimension $m+k$. Together with map

$$
\partial^{+} \times e: P \times_{R_{j}^{s}} \overline{\mathcal{M}}^{*}(j, i) \rightarrow R_{i}(H) \times V
$$

it gives a geometric chain of $R_{i}(H) \times V$. We define $\alpha(\kappa)((P, f))=\kappa \times$ $\alpha\left(\left(P \times_{R_{j}^{s}} \overline{\mathcal{M}}^{*}(j, i), \partial^{+} \times e\right)\right)$.

Using the fact that $\alpha$ is closed, we can show that it is a chain map, i.e.,

Lemma 3.2. We have $\delta \alpha-\alpha \delta=0$ 
Corollary 3.3. $\alpha$ induces an action of $[\alpha]$ of $H^{*}(V, Z)$ on the Bott-type Floer cohomology $H F^{*}(V, H)$.

Lemma 3.4. The action $[\alpha]$ is independent of $H$.

Theorem 1.4 follows from Corollary 3.3 and lemma 3.4.

When $H$ is nondegenerate, it follows from the definition that the extrinsic multiplication we just defined is the same as Floer's. When $H=0$, it is easy to check that it is indeed the quantum multiplication if we view $[\alpha]$ as an element of the quantum cohomology (cf. 1.5).

Corollary 3.5. The extrinsic multiplication is equivalent to the quantum multiplication.

\section{The intrinsic multiplication}

As we mentioned before, there is another way to define a multiplication between elements of the Floer cohomology; in terms of pseudo-holomorphic maps from a pair-pants to a symplectic manifold, whose ends approach to periodic orbits. Now we generalize this construction to the Bott-type case.

Let $T$ be a pair-pants, which has two outgoing ends $T_{1}^{+}, T_{2}^{+}$and one incoming end $T^{-}$. The incoming end is modeled by $S^{1} \times(-\infty, 0]$ and outgoing end is modeled by $S^{1} \times[0, \infty)$. We fix a parameterization for three ends. For any compatible almost complex structure $J$, we consider the smooth map $f: T \rightarrow V$ such that $f$ satisfies the equation

$$
d f(x)+J \cdot d f \cdot j_{T}(x)=\nu(x, f(x)), x \in T
$$

where $j_{T}$ denotes the conformal structure on $T$, and $\nu$ is an inhomogeneous term on $T \times V$, i.e., $\nu(x, y)$ is a linear map from the tangent space of $T$ at $x$ into the tangent space of $V$ at $y$ satisfying $\nu(x, y) \cdot j_{T, x}=-J_{y} \cdot \nu(x, y)$. Any such map $f$ is called a $(J, \nu)$-holomorphic map, or simply a $(J, \nu)$ map. Let $\mathcal{M}_{T}$ be the set of all $(J, \nu)$-maps. We will assume that our $\nu$ is given by $H$ near each end of $T$ as follows: if $x=(t, s)$ with $|s|$ sufficiently large, we have

$$
\nu(x, y)\left(\frac{\partial}{\partial t}\right)=\nabla_{y} H(t, y), \nu(x, y)\left(\frac{\partial}{\partial s}\right)=J \nabla_{y} H(t, y)
$$

Note that $j_{T}\left(\frac{\partial}{\partial s}\right)=\frac{\partial}{\partial t}$. By using the same argument as that in the proof of Lemma 2.1, one can show

Lemma 4.1. For every $u \in \mathcal{M}_{T}$, over any end of $T, u_{s}(t)=u(s, t)$ converges to $u_{ \pm \infty}(t) \in R(H)$, when $s \rightarrow \pm \infty$. If $H$ is of the Bott-type, $u_{s}$ converges exponentially to its limit, i.e., $\left|u_{s}-u_{ \pm \infty}\right|<C e^{-\delta|s|}$ for $s \geq|T|$. 
We will use $\partial_{+}^{1}, \partial_{+}^{2}, \partial_{-}$to denote the boundary value of ends $T_{1}^{+}, T_{2}^{+}, T^{-}$. By this lemma, we can divide the moduli space

$$
\mathcal{M}_{T}=\cup \mathcal{M}_{T}(k ; i, j)
$$

where

$$
\mathcal{M}_{T}(k ; i, j)=\left\{u \in \mathcal{M}_{T} \mid \partial_{+}^{1} u \in R_{i}, \partial_{+}^{2} u \in R_{j}, \partial_{-} u \in R_{k}\right\} .
$$

For generic $J, \nu$, the $\mathcal{M}_{T}(k ; i, j)$ are smooth manifolds of dimension $\operatorname{dim} R_{k}+k-i-j$, transverse to each other whenever they have the same target space. Again, by the same argument, we can show that the compactification $\overline{\mathcal{M}}_{T}(k ; i, j)$ is an abstract geometric chain. By abusing notation, we also use $C^{t}$ to denote the geometric chains transverse to the boundary maps of $\mathcal{M}_{T}(k ; i, j)$ and $\mathcal{M}(i, j)$. Now we are ready to define the intrinsic multiplication

$$
h: C_{t}^{m}(V, H) \otimes C_{t}^{k}(V, H) \rightarrow C_{t}^{m+k}(V, H)
$$

such that

$$
\delta(h(x \otimes y))=h\left(\delta x \otimes y+(-1)^{\operatorname{deg}(x)} x \otimes \delta y\right) .
$$

Let $x \in C_{t}^{m-i}\left(R_{i}, Z\right), y \in C_{t}^{k-j}\left(R_{j}, Z\right)$. Then

$$
h(x \otimes y) \in \sum_{r} C^{m+k-r}\left(R_{r}, Z\right) .
$$

If $h(x \otimes y)=\sum_{r \geq i+j} h^{r}(x \otimes y)$, where $h^{r}(x \otimes y) \in C_{t}^{m+k-r}\left(R_{r}, Z\right)$, then $h^{r}(x \otimes y) \in C_{t}^{m+k-r}\left(R_{r}, Z\right)$ is defined as follows:

For any $(P, f) \in C_{m+k-r}^{t}\left(R_{r}, Z\right)$,

$$
\left(P \times R_{r} \overline{\mathcal{M}}_{T}(r ; i, j), \partial_{+}^{1} \times \partial_{+}^{2}\right)
$$

is a geometric chain of degree $m+k-i-j$ in $R_{i} \times R_{j}$. We define

$$
h^{r}(x \otimes y)((P, f))=(x \times y)\left(\left(P \times_{R_{r}} \mathcal{M}_{T}(r ; i, j), \partial_{+}^{1} \times \partial_{+}^{2}\right)\right) .
$$

Clearly, $h$ preserves the filtration. It is straight forward to check

Lemma 4.2. $\delta h(x \otimes y)=h\left(\delta x \otimes y+(-1)^{\operatorname{deg}(x)} x \otimes \delta y\right)$.

Theorem 4.3. $h$ induces a multiplication on the Bott-type Floer cohomology $H F^{*}(V, H)$. Such a multiplication is independent of the hamiltonian $H$. We call it intrinsic multiplication and denote it by $x \star y$.

This is just Theorem 1.6. It follows from the definition that

Corollary 4.4. When $H=0$, the multiplication $h$ is the same as the quantum multiplication. 


\section{The quantum Massey product}

In $[\mathrm{F}]$, Fukaya defined a quantum Massey product and the quantum version of various secondary operations. Recall that Fukaya defined them by using moduli space of the grading flow lines of several Morse functions. In practice, it is very difficult to calculate such a quantized Massey product. For instance, it is hard to see if such a quantum product vanishes on a compact Kähler manifold. Therefore, it would be very important to define such a quantum Massey product in a different way. In this section, we will define the quantum Massey product in terms of our Bott-type Floer cohomology. Our construction is motivated by that of Fukaya. When $H$ is nondegenerate, it will give the Massey product for the ordinary Floer cohomology defined by Fukaya. A particular interesting case is $H=0$, where the definition doesn't rely on any hamiltonian or Morse function. A famous theorem of Deligne, Griffiths, Morgan and Sullivan says that the ordinary Massey product vanishes on a compact Kähler manifold. It looks likely from our definition that the quantum Massey product is zero on a compact Kähler manifold. The quantum Massey product appeared in $[\mathrm{BD}]$ for some representation spaces of flat bundles over Riemann surfaces. An interesting problem is whether or not one can quantize holomorphic torsions for Käher manifolds. We leave it for future research.

Let us define the quantum Massey product. Let $\alpha, \beta$ and $\gamma$ be three cocycles representing cohomology classes $[\alpha],[\beta]$ and $[\gamma]$ in $H^{*}(V, Z)$. Suppose that $[\alpha] \star[\beta]=0$ and $[\beta] \star[\gamma]=0$. Then by definition, there are cochains $x$ and $y$ such that $h(\alpha, \beta)=\delta x$ and $h(\beta, \gamma)=\delta y$, where $h(\cdot, \cdot)$ was defined in the last section by using a pair-pants. The coboundary of $h(x, \gamma)$ is $h(h(\alpha, \beta), \gamma)$, while the coboundary of $h(\alpha, y)$ is $(-1)^{\operatorname{deg} \alpha} h(\alpha, h(\beta, \gamma))$. These two coboundaries do not necessarily coincide, but they are cobordant to each other. In order to find this cobordism, we will first introduce a homomorphism $h_{3}$.

Let $\pi: S \mapsto[0,1]$ be a fibration such that $\pi^{-1}(u)$ is diffeomorphic to a sphere with 4 punctures for $0<u<1$ and the union of two $S^{2}$ 's transverse to each other for $u=0,1$. We denote by $p_{0}$ and $p_{1}$ the nodes in $\pi^{-1}(0)$ and $\pi^{-1}(1)$. Let $\sigma_{i}(i=1, \cdots, 4)$ be four disjoint sections of the fibration satisfying:

(1) $\sigma_{1}(0), \sigma_{2}(0)$ are in one connected component of $\pi^{-1}(0) \backslash p_{0}$;

(2) $\sigma_{3}(0), \sigma_{4}(0)$ are in one connected component of $\pi^{-1}(0) \backslash p_{0}$;

(3) $\sigma_{1}(1), \sigma_{3}(1)$ are in one connected component of $\pi^{-1}(1) \backslash p_{1}$;

(4) $\sigma_{2}(1), \sigma_{4}(1)$ are in one connected component of $\pi^{-1}(1) \backslash p_{1}$.

We put

$$
S^{\prime}=S \backslash\left\{p_{0}, p_{1}, \sigma_{1}([0,1]), \sigma_{2}([0,1]), \sigma_{3}([0,1]), \sigma_{4}([0,1])\right\}
$$


We assume that $S^{\prime}$ is modeled by $S^{1} \times[0, \infty) \times[0,1]$ near $\sigma_{i}([0,1])(i=$ $1,2,3)$ and by $S^{1} \times(-\infty, 0] \times[0,1]$ near $\sigma_{4}([0,1])$. Choose a generic inhomogenous term $\nu$ on $S^{\prime} \times V$ such that near each $\sigma_{i}([0,1])$, we have

$$
\nu(t, s, u, y)\left(\frac{\partial}{\partial t}\right)=\nabla_{y} H(t, y), \nu(t, s, u, y) \frac{\partial}{\partial s}=J(y) \nabla_{y} H(t, y)
$$

where $y \in V$ and $|s|$ is sufficient large, furthermore $\left.\nu\right|_{\pi^{-1}(0), \pi^{-1}(1)}$ is defined as in (4.2) near each node. Let $\mathcal{M}_{S}$ be the set of $(J, \nu)$-maps from some $\pi^{-1}(u)$ into $V$. As before, one can show that each $f \in \mathcal{M}_{S}$ converges exponentially to a point in some $R_{i}$. We shall use $\partial_{+}^{1} f, \partial_{+}^{2} f, \partial_{+}^{3} f$ and $\partial_{-} f$ to denote the limits at $\sigma_{1}(u), \sigma_{2}(u), \sigma_{3}(u)$ and $\sigma_{4}(u)$. Then

$$
\mathcal{M}_{S}=\bigcup \mathcal{M}\left(j ; i_{1}, i_{2}, i_{3}\right)
$$

where for any $f$ in $\mathcal{M}\left(j ; i_{1}, i_{2}, i_{3}\right), \partial_{+}^{p} f \in R_{i_{p}}$ and $\partial_{-} f \in R_{j}$. For a generic pair $(J, \nu), \mathcal{M}\left(j ; i_{1}, i_{2}, i_{3}\right)$ is a smooth, oriented manifold of dimension $\operatorname{dim} R_{j}+j-i_{1}-i_{2}-i_{3}+1$. Furthermore, $\partial_{ \pm}^{i}$ are transverse to each other whenever they have the same target space. By abusing notation, we again use $C_{t}^{*}\left(R_{i}, Z\right)$ to denote the complex of geometric chains transverse to all the boundary maps.

Lemma 5.1. The Gromov-Uhlenbeck compactification $\overline{\mathcal{M}}\left(j, i_{1}, i_{2}, i_{3}\right)$ of $\mathcal{M}\left(j ; i_{1}, i_{2}, i_{3}\right)$ is an abstract geometric chain.

The proof of this lemma is the same as that of Theorem 2.4.

We define a homomorphism

$$
h_{3}: C_{t}^{*}(V, H) \otimes C_{t}^{*}(V, H) \otimes C_{t}^{*}(V, H) \mapsto C_{t}^{*}(V, H)
$$

as follows: let $\beta_{l} \in C_{t}^{m_{l}-i_{l}}\left(R_{i_{l}}, Z\right)$, where $l=1,2,3$. Suppose that

$$
h_{3}\left(\beta_{1}, \beta_{2}, \beta_{3}\right)=\sum_{i} h_{3}^{i}\left(\beta_{1}, \beta_{2}, \beta_{3}\right)
$$

where $m=m_{1}+m_{2}+m_{3}$ and $h_{3}^{i}\left(\beta_{1}, \beta_{2}, \beta_{3}\right) \in C_{t}^{m-1-i}\left(R_{i}, Z\right)$. Let us define $h_{3}^{i}\left(\beta_{1}, \beta_{2}, \beta_{3}\right)$ as follows: for any $(P, f)$ in $C_{t}^{m-1-i}\left(R_{i}, Z\right)$, by Lemma 5.1, the fiber product $P \times_{R_{j}} \overline{\mathcal{M}}\left(j ; i_{1}, i_{2}, i_{3}\right)$ is an abstract geometric chain. Together with the boundary map $\partial_{+}^{1} \times \partial_{+}^{2} \times \partial_{+}^{3}$, this gives rise to a geometric chain in $R_{i_{1}} \times R_{i_{2}} \times R_{i_{3}}$ of degree $m-i_{1}-i_{2}-i_{3}$. Then

$h_{3}^{i}\left(\beta_{1}, \beta_{2}, \beta_{3}\right)((P, f))=\beta_{1} \otimes \beta_{2} \otimes \beta_{3}\left(P \times_{R_{j}} \overline{\mathcal{M}}\left(j ; i_{1}, i_{2}, i_{3}\right), \partial_{+}^{1} \times \partial_{+}^{2} \times \partial_{+}^{3}\right)$

Now we return to the definition of the quantum Massey product. Let $\alpha, \beta$ and $\gamma$ be the cocycles as above. One can easily show

$$
\delta h_{3}(\alpha, \beta, \gamma)=-h(h(\alpha, \beta), \gamma)+(-1)^{\operatorname{deg} \alpha} h(\alpha, H(\beta, \gamma))
$$


Therefore, we have

$$
\delta\left(\eta^{2}(x, \gamma)+(-1)^{\operatorname{deg} \alpha-1} \eta^{2}(\alpha, y)+h_{3}(\alpha, \beta, \gamma)\right)=0
$$

Definition 5.2. The Massey product $m([\alpha],[\beta],[\gamma])$ of $[\alpha],[\beta],[\gamma]$ is defined to be the cohomology class in

$$
H^{\operatorname{deg} \alpha+\operatorname{deg} \beta+\operatorname{deg} \gamma-1}(V, Z) /\left(\alpha \star H^{*}(V, Z)+\gamma \star H^{*}(V, Z)\right)
$$

represented by the cocycle

$$
m(\alpha, \beta, \gamma)=h(x, \gamma)+(-1)^{\operatorname{deg} \alpha-1} h(\alpha, y)+h_{3}(\alpha, \beta, \gamma)
$$

where $h(\alpha, \beta)=\delta x$ and $h(\beta, \gamma)=\delta y$. Note that the quotient by $\alpha \star$ $H^{*}(V, Z)+\gamma \star H^{*}(V, Z)$ reflects the fact that there are no canonical choices of $x, y$.

One can show that this quantum Massey product is independent of the hamiltonian function $H$. A particularly interesting case is when $H$ is identically zero. If the moduli space of holomorphic maps from $S^{2}$ into $V$ is reasonably good, the homomorphism $h_{3}$ can be defined by using only holomorphic maps; consequently, the quantum Massey product can be defined by using only holomorphic maps. This indicates that the quantum Massey product is related to the Massey product on the moduli spaces of holomorphic maps and, consequently, the vanishing of the quantum Massey product on a compact Kähler manifold.

More generally, we can start with a Riemann sphere $T_{k}$ with $k+1$ marked points which are on the equator. Furthermore, we first fix three points and allow the rest of the marked points to move on the equator in a suitable order. Then, we remove the marked points and regard a neighborhood of each marked points as an infinite cylinder. Here, we orient in such a way that first $k-1$ cylinders are outgoing and last one is incoming. As we described in the last section, we choose an inhomogeous term $\nu$ which is given by the hamiltonian function $H$ in a neighborhood of each marked point, allowing us to consider the moduli space $\mathcal{M}_{T_{k}}$ of $(J, \nu)$-maps defined. Then one can define $h_{k}$ in the exactly same way as we defined $h_{3}$. For $\beta_{i} \in C_{t}^{m_{i}-p_{i}}\left(R_{p_{i}}, Z\right)$ for $1 \leq i \leq k$, we have

$$
h_{k}\left(\beta_{1}, \cdots, \beta_{k}\right)=\sum h_{k}^{i}\left(\beta_{1}, \cdots, \beta_{k}\right), h_{k}^{i}\left(\beta_{1}, \cdots, \beta_{k}\right) \in C_{t}^{m+2-k-i}\left(R_{i}, Z\right)
$$

where $m=\sum m_{i}$. More precisely, if $(P, f) \in C_{m+2-k-i}^{t}\left(R_{i}, Z\right)$, then we have

$$
h_{k}^{i}\left(\beta_{1}, \cdots, \beta_{k}\right)((P, f))=\beta_{1} \otimes \cdots \otimes \beta_{k}\left(\left(P \times_{R_{i}} \overline{\mathcal{M}}\left(i ; p_{1}, \cdots, p_{k}\right), \prod_{p_{j}} \partial_{p_{j}}^{+}\right)\right) .
$$


It is not hard to see that $h_{1}=\delta, h_{2}=h$. Moreover,

$$
\delta h_{k}\left(\beta_{1}, \cdots, \beta_{k}\right)=\sum_{i<j} \epsilon h_{k-j+i+1}\left(\beta_{1}, \cdots, h_{j-i}\left(\beta_{i+1}, \beta_{j}\right), \cdots, \beta_{k}\right),
$$

where $\epsilon=(-1)^{(j-i)\left(\operatorname{deg} \beta_{1}+\cdots+\operatorname{deg} \beta_{i}\right)}$. Then one can define more general multiplications by using these homomorphisms $h_{k}$ (cf. [Fu]).

Remark. In our basic model $T_{k}$, we can also fix all the marked points. Then the resulting multiplication is just the higher quantum product by the composition law the authors proved in [RT].

\section{References}

[AB] D. Austin and P. Braam, Equivariant Floer (co)-homology and gluing Donaldson polynomials, preprint.

[BR] M. Batz and J. Rade, Products and relations in symplectic Floer homology, in preparation.

[BD] P. Braam and S. Donaldson, Fukaya-Floer homology and gluing formula for polynomial invariants, preprint.

[CZ] C. Conley and E. Zehnder, The Birkhoff-Lewis fixed point theorem and a conjecture of V.I. Arnold, Invent. Math. 73 (1983), 33-49.

[F] A. Floer, Symplectic fixed points and holomorphic spheres, Comm. Math. Phys. 120 (1989), 575-611.

[Fu] K. Fukaya, Floer homology for connected sum, preprint.

[Fu1] , Morse homotopy and its quantization, preprint.

[HS] H. Hofer and D. Salamon, Floer homology and Novikov rings, preprint.

[M] T. Mrowka, A local Mayer-Vietoris principal for Yang-Mills moduli spaces, PhD thesis, Berkeley, 1989.

[MMR] J. Morgan, T. Mrowka and D. Ruberman, The $L^{2}$-moduli space and a vanishing theorem for Donaldson polynomial invariants, Monographs in Geom. and Topo., International Press.

[OV] K. Ono and L. Van, Cup-length estimate for symplectic fixed points, preprint.

[P] S. Piunikhin, Quantum and Floer cohomology have the same ring structure, preprint.

[RT] Y. Ruan and G. Tian, A mathematical theory of quantum cohomology, to appear in JDG.

[S] V. Sadov, On the equivalence of Floer's and quantum cohomology, preprint, Harvard University HUTP-93/A027 (1993).

Dept. of Math., Univ. of Utah, Salt Lake City, UT 84112

E-mail address: ruan@math.utah.edu

Courant Institute of Math. Sci., 251 Mercer st., New York University, NY 10012

E-mail address: tiang@cims20.nyu.edu 